\title{
Should We Routinely Screen for Warfarin Gene Polymorphism in Patients with Coumadin Overdose?
}

Enes Elvin Gül, Halil İbrahim Erdoğan, Mehmet Yazıcı

Department of Cardiology, Selçuk University Meram School of Medicine, Konya, Turkey

\begin{abstract}
Genetic polymorphisms can affect an individual's response to the pharmacological agents. Warfarin has a narrow therapeutic range and dose arrangement may vary from individual to individual. An insufficient dose may fail to prevent thromboembolism and overdose may increase the risk of bleeding. Increasing evidence suggests that genetic variation of CYP2C9 and VKORC1 greatly influences effective warfarin dosing (1). In the present case report, frequently occurring warfarin toxicity was caused by a genetic mutation in the patient with mitral valve replacement.

Keywords: warfarin, genetic, CYP2C9, VKORC1
\end{abstract}

\section{Case Report}

A 45 year-old female patient applied to the cardiology clinic with the complaint of nose-bleeding. The patient had a history of mitral valve re-placement one year ago and she had been started on warfarin ( $5 \mathrm{mg} /$ day). The patient had been followed up for recurrently developing warfarin toxicity in different clinics. When she applied to our clinic with the INR (International Normalized Ratio) value of 8.9, she was hospitalized due to warfarin toxicity. The patient, who had no active bleeding, was given 2 units of fresh frozen plasma (FFP). After FFP application, the INR value regressed to 3.5. The patient, whose INR value was 2.3 in the follow-up, was restarted on warfarin $(2.5 \mathrm{mg})$ and was discharged with recommendations. After one week, the patient applied to the emergency unit due to active nose bleeding and because examinations revealed an INR value of 14.7. She was hospitalized in our clinic for warfarin toxicity again. The patient was given $20 \mathrm{mg}$ intravenous vitamin $\mathrm{K}$ and 2 units of FFP. The INR value in the control examination was 3.07 and the complaint of active bleeding regressed. Warfarin gene polymorphism was studied in order to adjust the dose of warfarin because the patient frequently had warfarin toxicity. Genetic testing revealed heterozygote mutation in the CYP2C9*2 C430T region and homozygote mutation in the VKORC1 C1173T and G1639A regions. The patient, who was found to have warfarin resistance and increased sensitivity, was discharged by recommending a quarter dose of warfarin for 2 days in a week and to visit the hospital for INR control one week later.

\section{Discussion}

Warfarin is the specific inhibitor of vitamin $\mathrm{K}$ epoxide reductase (VKOR), which is coded by the VKORC1 gene. Warfarin shows its anticoagulant effects by preventing the competence of VKORC1 in the production of vitamin $\mathrm{K}$ reduced from the epoxide form. Functional abnormalities in VKORC1 are also known as the resistance to coumarin-type anticoagulant drugs (warfarin resistance) $(1,2)$. Moreover, due to the mutation in VKORC1, vitamin $\mathrm{K}$ cannot be activated through hydroxylation and no coagulation occurs. Genetic polymorphisms in VKORC1 clearly affect the dose of warfarin and cause undesirable bleeding events (3). In a study conducted on this condition, the data of 110 patients that had severe bleeding attacks under warfarin treatment and 220 control cases without any bleeding, despite receiving a similar treatment, were compared. The patients were specifically examined in terms of VKORC1 C1173T polymorphism and an increase was observed in the risk of bleeding in patients with existent mutation (4). Coumarin derivatives were used in this study (4).

Warfarin is a racemic mixture and it is metabolized via cytochrome enzymes in the liver. In stable conditions, S-warfarin constitutes $60 \%-70 \%$ of the anticoagulant response and the R-enantiomer constitutes $30 \%-40 \%$. Primarily, S-warfarin is metabolized by CYP2C9 and R-warfarin by CYP3A4, CYP1A2, and CYP1A1. The genetic variations of CYP2C9, CYP3A4, CYP1A2, and CYP1A1 lead to individual deviations in the potentially effective dose of warfarin, and the isomer that is frequently studied among these cytochromes is CYP2C9 (5).

Correspondence to: Enes Elvin Gül e-mail: elvin_salamov@yahoo.com 
Currently, more than 50 variants of CYP2C9 have been defined, but CYP2C9*2 and CYP2C9*3, which are seen more often, are important with regard to their effects on the dose of warfarin (3). The direct relationship between the CYP2C9 genotype and anticoagulant (or bleeding) effects was firstly reported by Higashi et al. (6). Subsequently, from a systematic meta-analysis, it was emphasized that a lower maintenance dose of warfarin was needed in patients with the CYP2C9*2 or CYP2C9*3 allele (7).

In our case, mutations were detected both in the CYP2C9*2 C430T region and in the VKORC1 C1173 T and G1639A regions. That is to say, our patient was prone to bleeding because of metabolism impairment and also in vitamin $\mathrm{K}$ activation. There were probably two causes of bleeding in our patient: Firstly, mutation in CYP2C9 prevented inactivation of warfarin and consequently, led to bleeding.
Secondly, impairment in the activation of vitamin $\mathrm{K}$ via hydroxylation due to mutation in VKORC1 inhibited the formation of coagulation and caused bleeding. Mutation in both metabolic pathways increased the risk of bleeding and led to bleeding by causing recurring warfarin toxicity in our patient. In this case, the only application that can be performed is serious reduction in the dose of warfarin (3). A schematic picture is presented for understanding warfarin metabolism and gene polymorphism (Figure 1).

In conclusion, this study emphasizes that genetic polymorphism must be studied for a patient having a history of mitral valve replacement and bleeding secondary to warfarin toxicity, and adjustment must be done according to the results of that study. We strongly recommend our colleagues encountering similar situations to perform warfarin polymorphism analysis.

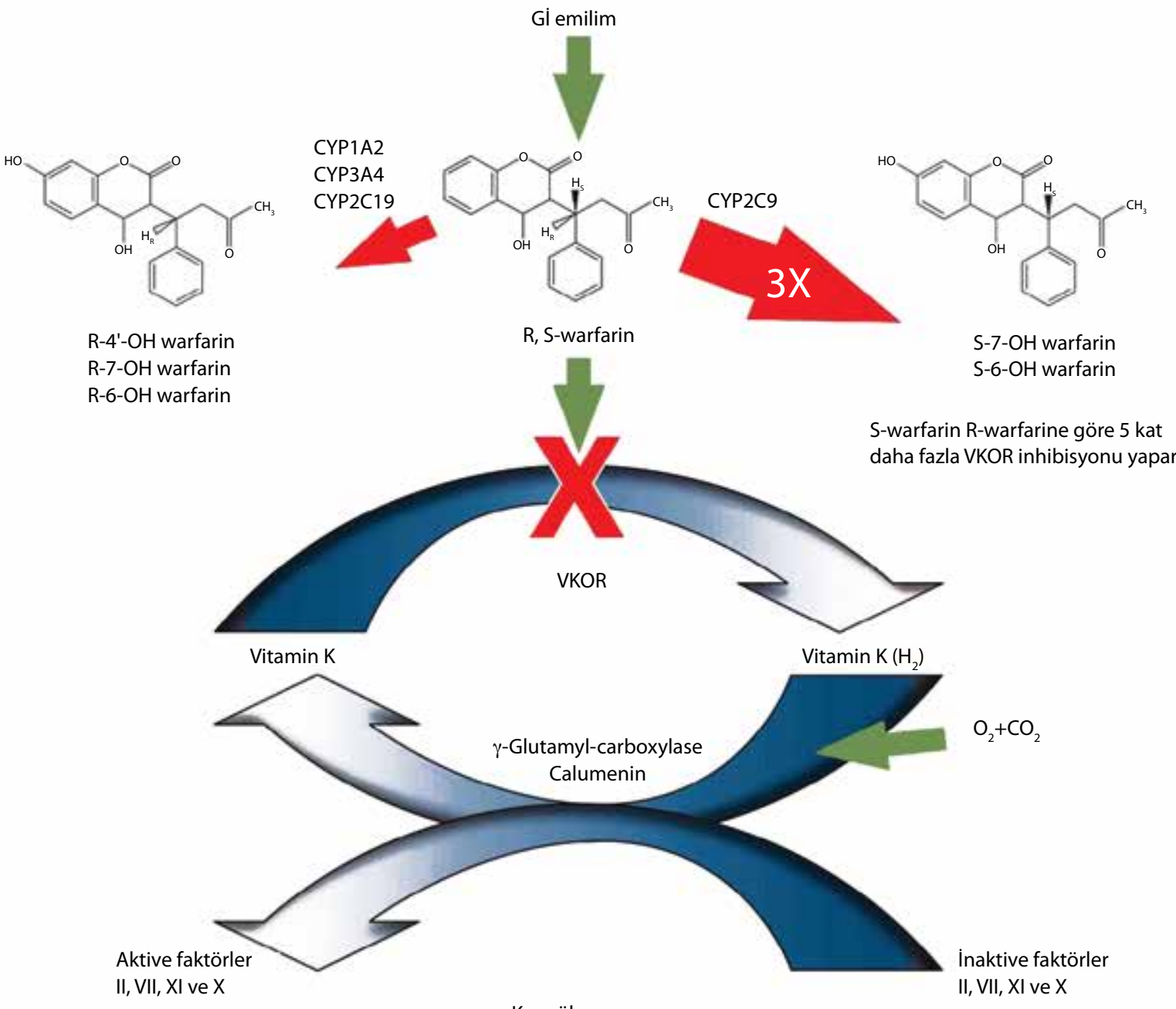

Koagülasyon

Figure 1. The coagulation cascade is inhibited by warfarin. Warfarin has two enantiomers (R-warfarin and S-warfarin), and it is absorbed from the gastrointestinal (GI) tract at the rate of 93\%. Warfarin is metabolized by cytochromes (often by CYP2C9) in the liver. CYP2C9 selectively performs hydroxylation of S-warfarin and converts it into inactive metabolites. CYP1A2, CYP3A4, and CYP2C19, which are other isomers of cytochrome P450, selectively perform hydroxylation of R-warfarin and converts it into inactive metabolites. S-warfarin is metabolized at a rate three times higher than that of R-warfarin. The Vitamin-K epoxide reductase (VKOR) enzyme hydroxylates vitamin $\mathrm{K}$ and it converts it into active vitamin $\mathrm{K}(\mathrm{Vit}-\mathrm{K}(\mathrm{H} 2)$ ). Active vitamin $\mathrm{K}$ activates inactive coagulation factors (factor II, $\mathrm{VII}, \mathrm{XI}$, and $\mathrm{X}$ ) through carboxylation. Moreover, S-warfarin inhibits vitamin K epoxide reductase (VKOR) 5 times more potently than R-warfarin. 


\section{References}

1. D'Andrea G, D'Ambrosio R, Margaglione M. Oral anticoagulants: pharmacogenetics relationship between genetic and non-genetic factors. Blood Rev 2008; 22:127-40.

2. Gage BF, Lesko LJ. Pharmacogenetics of warfarin: regulatory, scientific, and clinical issues. J Thromb Thrombolysis 2008; 25: 45-51. [CrossRef]

3. Yin T, Miyata T. Warfarin dose and the pharmacogenomics of CYP2C9 and VKORC1 - rationale and perspectives. Thromb Res 2007; 120: 1-10. [CrossRef]
4. Reitsma PH, van der Heijden JF, Groot AP, Rosendaal FR, Büller HR. A C1173T dimorphism in the VKORC1 gene determines coumarin sensitivity and bleeding risk. PLoS Med 2005; 2: e312. [CrossRef]

5. Redman AR. Implications of cytochrome P450 2C9 polymorphism on warfarin metabolism and dosing. Pharmacotherapy 2001; 21:235-42. [CrossRef]

6. Higashi MK, Veenstra DL, Kondo LM, Wittkowsky AK, Srinouanprachanh SL, Farin FM, et al. Association between CYP2C9 genetic variants and anticoagulation-related outcomes during warfarin therapy. JAMA 2002; 287: 1690-8. [CrossRef]

7. Sanderson S, Emery J, Higgins J. CYP2C9 gene variants, drug dose, and bleeding risk in warfarin-treated patients: a HuGEnet systematic review and meta-analysis. Genet Med 2005; 7: 97-104. [CrossRef] 